\title{
Efficiency of Functional Brain Networks and Intellectual Performance
}

\author{
Martijn P. van den Heuvel, ${ }^{1}$ Cornelis J. Stam, ${ }^{2}$ René S. Kahn, ${ }^{1}$ Hilleke E. Hulshoff Pol ${ }^{1}$ \\ ${ }^{1}$ Department of Psychiatry, Rudolf Magnus Institute of Neuroscience, University Medical Center Utrecht, 3508 GA Utrecht, The Netherlands, and \\ ${ }^{2}$ Department of Clinical Neurophysiology, VU University Medical Center, 1007 MB Amsterdam, The Netherlands
}

Our brain is a complex network in which information is continuously processed and transported between spatially distributed but functionally linked regions. Recent studies have shown that the functional connections of the brain network are organized in a highly efficient small-world manner, indicating a high level of local neighborhood clustering, together with the existence of more long-distance connections that ensure a high level of global communication efficiency within the overall network. Such an efficient network architecture of our functional brain raises the question of a possible association between how efficiently the regions of our brain are functionally connected and our level of intelligence. Examining the overall organization of the brain network using graph analysis, we show a strong negative association between the normalized characteristic path length $\lambda$ of the resting-state brain network and intelligence quotient (IQ). This suggests that human intellectual performance is likely to be related to how efficiently our brain integrates information between multiple brain regions. Most pronounced effects between normalized path length and IQ were found in frontal and parietal regions. Our findings indicate a strong positive association between the global efficiency of functional brain networks and intellectual performance.

\section{Introduction}

Our brain is a complex network of interconnected regions (Sporns et al., 2004; Achard et al., 2006; Stam and Reijneveld, 2007; Hagmann et al., 2008; van den Heuvel et al., 2008c, 2009; Buckner et al., 2009; Honey et al., 2009). Within this brain network, information is constantly processed and integrated between specialized, spatially distributed but functionally linked brain regions with coherent temporal dynamics (Sporns et al., 2000). This integration of information is a never-ending process that goes on even when we are at rest (Raichle et al., 2001; Greicius et al., 2003; Damoiseaux et al., 2006; van den Heuvel et al., 2008a). This ongoing integration of information enables us to evaluate the world around us and to respond quickly and flexibly to complex situations. Recent studies have shown that the functional connections of the brain network are organized in a highly efficient small-world manner (Sporns et al., 2004; Stam, 2004; Eguíluz et al., 2005; Achard et al., 2006; van den Heuvel et al., 2008b). A small-world organization of the brain network suggests a high level of local neighborhood clustering, responsible for efficient local information processing, together with the existence of several long-distance connections that ensure a high level of global communication efficiency across the network and integration of information between the different regions of the brain (Watts and Strogatz, 1998; Latora and Marchiori, 2001; Stam and Reijneveld, 2007; Bullmore and Sporns, 2009). Such an efficient

\footnotetext{
Received March 26, 2009; revised April 29, 2009; accepted May 6, 2009.

We thank Judith Luigjes and Anne Klomp for their assistance in the data collection.

Correspondence should be addressed to Martijn P. van den Heuvel, Department of Psychiatry, Rudolf Magnus Institute of Neuroscience, University Medical Center Utrecht, Heidelberglaan 100, P.0. Box 85500, 3508 GA Utrecht, The Netherlands. E-mail: m.p.vandenheuvel@umcutrecht.nl.

DOI:10.1523/JNEUROSCI.1443-09.2009

Copyright $\odot 2009$ Society for Neuroscience $\quad$ 0270-6474/09/297619-06\$15.00/0
}

organization of our brain network raises the question of a possible relationship between how efficiently the functional connections of our brain are placed and individual differences in intelligence. Neuroimaging studies have linked intelligence to the developmental course of specific high-order brain regions (Shaw et al., 2006), total brain volume and focal brain structure (Thompson et al., 2001; Haier et al., 2004; Colom et al., 2006; Hulshoff Pol et al., 2006; Choi et al., 2008), microstructural organization of white matter (Chiang et al., 2009), and the functional dynamics of specific high cognitive brain regions (Duncan et al., 2000; Gray et al., 2003; Choi et al., 2008; Song et al., 2008). However, it remains unknown whether, and if so, how intellectual performance is related to the overall connectivity network architecture of our brain.

\section{Materials and Methods}

Subjects. Nineteen healthy subjects without a psychiatric history (age mean/SD: 29/7.8; gender: 14 male, 5 female) (Table 1) participated in this study after providing written informed consent as approved by the medical ethics committee for research in humans (METC) of the University Medical Center Utrecht, The Netherlands.

Intelligence scores. The level of intellectual performance of the participants was measured with the Dutch version of the Wechsler Adult Intelligence Scale III (WAIS-III) test. The WAIS test gives a standardized full-scale intelligence quotient, in this paper referred to as IQ, based on a number of subtests that assess the level of verbal [verbal IQ (VIQ)] and nonverbal knowledge and reasoning [performance IQ (PIQ)] of the participants. Table 1 describes the IQ data, and Table 2 describes the correlations between the IQ scores.

Data acquisition. The functional connections of the brain network were examined by measuring the correlations between the spontaneous brain signals of the different regions of the brain during rest (Aertsen et al., 1989; Biswal et al., 1995; Salvador et al., 2005; Achard et al., 2006). Resting-state functional magnetic resonance imaging (fMRI) time series 
Table 1. Demographic and IQ data

\begin{tabular}{lc}
\hline Category & Data \\
\hline Gender (male/female) & $14 / 5$ \\
Age, years & $29 \pm 7.8$ \\
Full-scale IQ & $121 \pm 11.9$ \\
Verbal IQ & $121 \pm 9.5$ \\
Verbal comprehension index & $119 \pm 9.5$ \\
Working memory index & $117 \pm 11.9$ \\
Performance IQ & $117 \pm 12.6$ \\
Perceptual organization index & $115 \pm 11.2$ \\
Processing speed index & $112 \pm 15.1$ \\
\hline
\end{tabular}

Age and $\mathrm{IQ}$ scores are displayed as mean \pm SD.

Table 2. Correlations between IQ scores

\begin{tabular}{lllllll}
\hline & FIQ & VIQ & PIQ & VCl & WMI & POI \\
\hline VIQ & 0.91 & & & & & \\
PIQ & 0.91 & 0.66 & & & & \\
VCI & 0.75 & 0.88 & 0.52 & & & \\
WMI & 0.72 & 0.67 & 0.61 & 0.36 & & \\
POI & 0.78 & 0.57 & 0.84 & 0.53 & 0.51 & \\
PSI & 0.63 & 0.53 & 0.65 & 0.41 & 0.39 & 0.40 \\
\hline
\end{tabular}

VCI, Verbal comprehension index; WMI, working memory index; PSI, processing speed index.

of each participant was acquired for a period of 8 min on a 3 tesla magnetic resonance imaging scanner [three-dimensional PRESTO, repetition time/echo time $22 \mathrm{~ms} / 32 \mathrm{~ms}$, flip angle $9^{\circ}$; SENSE $\mathrm{p} / \mathrm{s} 2 / 2$; a dynamic scan time of $0.5 \mathrm{~s}, 1000$ time frames; field of view $256 \times 256 \mathrm{~mm}$, voxel size $4 \times 4 \times 4 \mathrm{~mm}$, 32 slices covering whole brain (supplemental material, available at www.jneurosci.org)].

Preprocessing. Before the graph analysis, the fMRI data were preprocessed (supplemental material, available at www.jneurosci.org). Restingstate fMRI time series were realigned to correct for possible small head movements and coregistered with the T1 image, for anatomical overlap. The $\mathrm{T} 1$ and realigned resting-state time series were normalized to standard space, using the normalization parameters of the T1 image. Finally, the resting-state time series were bandpass filtered $(0.01-0.1 \mathrm{~Hz})$.

Graph analysis. The organization of the functional brain network was examined using graph theory (Achard et al., 2006; Stam and Reijneveld, 2007; Bullmore and Sporns, 2009), as validated earlier (van den Heuvel et al., 2008b) (supplemental material, available at www.jneurosci.org). In summary, individual functional brain networks were formed out of all the cortical and subcortical brain voxels ( $\sim 9500$ voxels, called nodes) with connections between all functionally linked voxels. The level of functional connectivity between any two voxels $i$ and $j$ was defined as the zero-lag correlation between their voxelwise resting-state blood oxygen level-dependent time series, and voxel $i$ and $j$ were defined as functionally linked when their zero-lag correlation reached above a threshold $T$ (ranging from 0.05 to 0.5) (Biswal et al., 1995; Achard et al., 2006; van den Heuvel et al., 2008a,b) (supplemental Fig. 1, available at www. jneurosci.org as supplemental material). From these functional brain networks, a number of key characteristics that describe the overall organization of a network were computed, including the clustering coefficient $C$ and characteristic path length $L$ (Watts and Strogatz, 1998). The clustering coefficient $C$ is given by the ratio between the number of connections between the direct neighbors of a node and the total number of possible connections between these neighbors and provides information about the level of local connectedness within a network. In addition, the characteristic path length $L$ of a network gives the average number of connections that have to be crossed to travel from each node to every other node in the network and provides information about the level of global communication efficiency of a network.

Networks with a small-world organization have a clustering coefficient $C$ that is much higher than the clustering coefficient of a comparable random organized network, but still with a short characteristic path length $L$ that is similar to that of an equivalent random organized network (Watts and Strogatz, 1998). Formally, small-world networks show a ratio $\gamma$ defined as $C / C^{\text {random }}$ of $\gg 1$ and a ratio $\lambda$ defined as $L / L^{\text {random }}$ of $\sim 1$, with $C^{\text {random }}$ and $L^{\text {random }}$ the clustering coefficient and characteristic path length of a random organized network of similar size (Watts and Strogatz, 1998; Sporns et al., 2004). $C^{\text {random }}$ and $L^{\text {random }}$ were computed as the average clustering coefficient and characteristic path length of a set of $h$ random graphs with a comparable total degree and degree distribution as that of the examined functional connectivity graph (supplemental material, available at www.jneurosci.org). $h$ was set to 20 for $T \geq 0.40$, 10 for $0.20 \leq T<0.40$, and to 5 for $T<0.20$, for computational reasons. Together, $\gamma$ and $\lambda$ provide important information about the level of local and global connectivity efficiency of a network. A high $\gamma$ reflects a high level of local neighborhood clustering within a network, and a short normalized travel distance $\lambda$ expresses a high level of global communication efficiency within a network (Watts and Strogatz, 1998; Sporns et al., 2004; Bullmore and Sporns, 2009).

Association between network organization and intelligence. To examine the relationship between the organization of the brain network and intelligence, the computed $\lambda$ and $\gamma$ values of the individual brain networks were correlated with the measured full-scale IQ scores of the participants. In addition, to examine whether intelligence is related to the overall number of connections of the brain network, the total number of connections $k$ was also correlated with the individual IQ scores.

To point out which specific brain regions have the strongest association between global network organization and IQ, an exploratory analysis was conducted, in which the normalized path length of each individual node (i.e., voxel) was correlated with IQ separately (supplemental material, available at www.jneurosci.org). The individual normalized path length of a node reflects how close this node is connected to the other nodes of the network, providing information about how efficiently this node is connected to the rest of the network. First, for each individual dataset, the normalized path length of each node was computed. Second, for all voxels separately, the normalized path length of voxel $i$ was correlated with the measured IQ scores over the group of subjects. This resulted in a correlation-coefficient map indicating which voxels showed a significant association between the full-scale IQ scores and normalized path length. An exploratory threshold of 0.05 (uncorrected for multiple comparisons) was used to mark brain regions that showed a significant association between their normalized path length and IQ.

\section{Results}

The functional brain networks showed a clear small-world organization for $0.3 \leq T \leq 0.5$ (Fig. $1 a-d$ ), expressed by $L \approx L^{\text {random }}$ and $\lambda \approx 1$ for $T \leq 0.5$ and $C \gg C^{\text {random }}$ and $\gamma \gg 1$ for $T \geq 0.3$ (one-sample $t$ test, all $p<\alpha$ of 0.01 , Bonferroni corrected for multiple comparisons of $T, \mathrm{df}=18$ ), indicating a small-world organization (Sporns et al., 2004; Stam, 2004; Achard et al., 2006; van den Heuvel et al., 2008b).

Next, the association between brain network organization and individual variation in intelligence was examined by correlating the full-scale IQ scores with the $\lambda$ and $\gamma$ values of the individual brain networks within the clear small-world range of $0.3 \leq T \leq$ 0.5 . For higher $T$, a significant negative association was found between the normalized characteristic path length $\lambda$ and IQ [linear regression, $T=0.45, r=-0.54, p=0.010, \mathrm{df}=18$ (Fig. $2 a)$; $T=0.5, r=-0.57, p=0.017$, df $=18$ (Fig. $2 b$ ), which is significant considering an $\alpha$ of 0.05 after Bonferroni correction for multiple comparisons of $T$; regressions corrected for age] (Fig. 2a,b; supplemental Fig. $3 a-c$, available at www.jneurosci. org as supplemental material). It is important to note that the observed association between $\lambda$ and IQ could not be related to possible variation in overall connectivity, as the IQ scores showed no correlation with the total number of connections $k$ of the brain network (supplemental Fig. $3 g-i$, available at www.jneurosci.org as supplemental material) or the distribution of $k$. No significant correlation was found between the clustering coefficient $\gamma$ and IQ (Fig. 2c,d). 

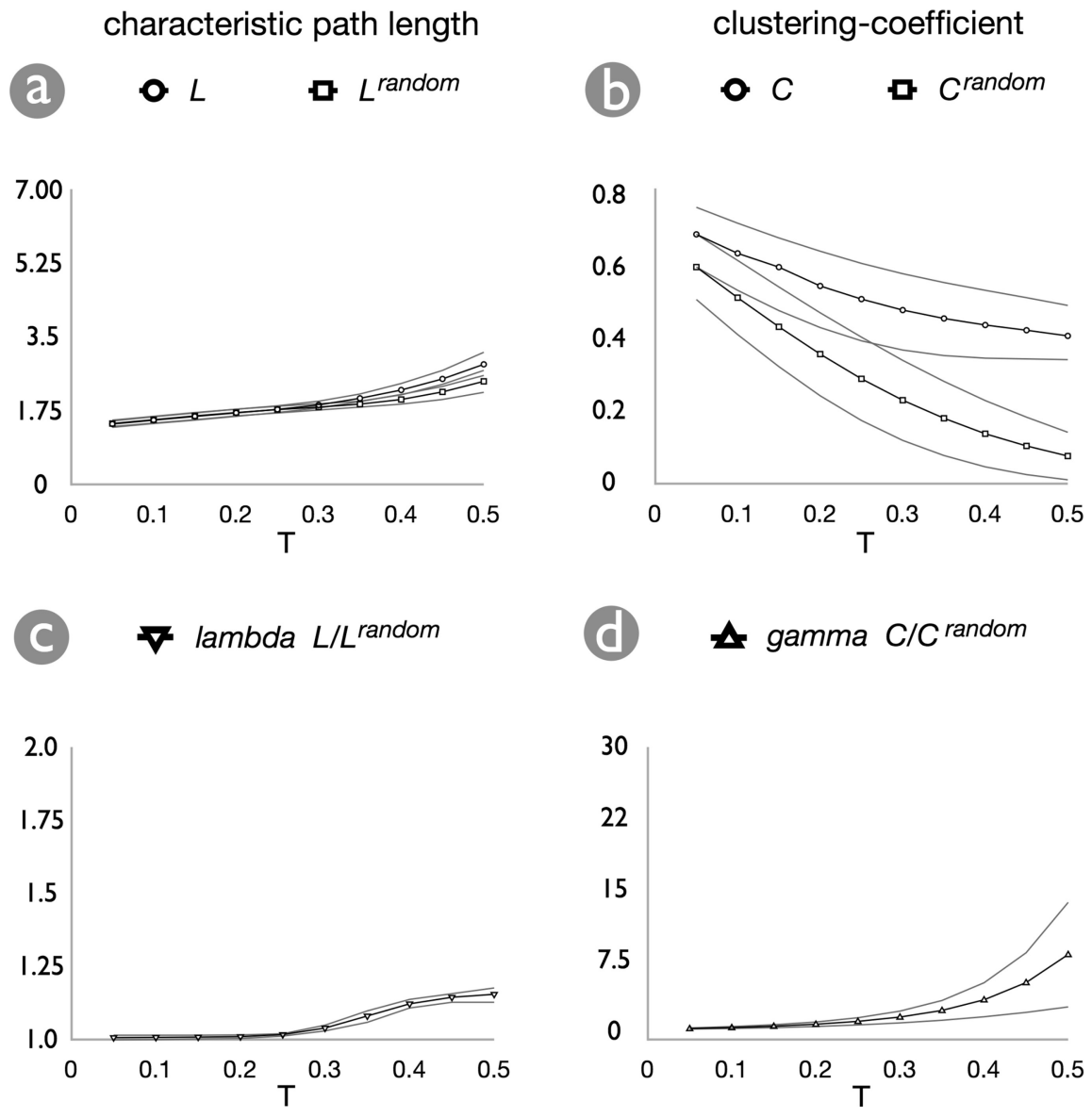

Figure 1. $\quad \boldsymbol{a}, \boldsymbol{b}$, The results of the group averaged characteristic path length $L$ and $L^{\text {random }}(\boldsymbol{a})$ and the results of clustering coefficient $C$ and $C^{\text {andom }}(\boldsymbol{b})$ for cutoff thresholds $0.05 \leq T \leq 0.5 .^{\text {random }}$ and $C^{\text {random }}$ reflect the characteristic path length and clustering coefficient of a comparable random network. $L$ was found to be $\sim L^{\text {random }}$ for $T \leq 0.5$, and (was found to be $>$ C random for $T \geq 0.3$ (2-sample $t$ test, $\mathrm{df}=36$, all $p<\alpha$ of 0.01, Bonferroni corrected for multiple comparisons of $T$ ). $\boldsymbol{c}, \boldsymbol{d}$, Matching $\lambda$ $\left(L / L^{\text {random }}\right)$ and $\gamma\left(C / C^{\text {random }}\right)$ values for all $T$. The functional brain networks showed a clear small-world organization, expressed by $\lambda \approx 1$ (c) and $\gamma \gg 1$ (d) for $T \geq 0.3$ (1-sample $t$ test, $\mathrm{df}=18$, all $p<\alpha$ of 0.01 , Bonferroni corrected for multiple comparisons of $T$ ).

To further probe which specific brain regions have the strongest association between global network organization and IQ, the normalized path length of each individual node (i.e., voxel) was correlated with the full-scale IQ scores separately. Figure 3 shows the correlation coefficients of those voxels that showed a significant correlation between their normalized path length and IQ (linear regression, $\mathrm{df}=18, p<0.05$ uncorrected for multiple comparisons, corrected for age). The most prominent effects between full-scale IQ and normalized path length were found in medial prefrontal gyrus (Brodmann area 9/10, $r=-0.75, p=$ $0.001, \mathrm{df}=18$, yellow box), precuneus/posterior cingulate gyrus (BA 7/31, $r=-0.55, p=0.014$, $\mathrm{df}=18$, orange box), and bilateral inferior parietal regions (BA 39/40, right inferior parietal: $r=-0.72, p=0.001, \mathrm{df}=18$ depicted in red box; left inferior parietal: $r=-0.68, p=0.001, \mathrm{df}=18)$. Also regions overlapping left superior temporal (BA 22/40, $r=-0.69, p=$ 0.014 , df $=18$ ) and left inferior frontal gyrus (BA 44/45, $r=$ $-0.68, p=0.012, \mathrm{df}=18$ ) showed a significant negative correlation between normalized path length and full-scale IQ.

\section{Discussion}

The main finding of this study is the existence of a strong association between the level of global communication efficiency of the functional brain network and intellectual performance. Our re- sults show that the normalized characteristic path length $\lambda$ of functional brain networks is strongly negatively associated with full-scale IQ (Fig. $2 a, b$ ). The characteristic path length $\lambda$ indicates how close the nodes of a network are connected globally, and the negative association between $\lambda$ and IQ suggests a strong positive association between the level of global communication efficiency within the brain network and general intelligence. In other words, more efficiently functionally connected brains show a higher level of intellectual performance.

The normalized clustering coefficient $\gamma$ and the number of connections $k$ (supplemental Fig. 3, available at www.jneurosci.org as supplemental material) were found not to be significantly associated with IQ, suggesting that intelligence is not directly related to the level of local information processing or to the total number of functional connections of the brain network. The strong association between $\lambda$ and IQ suggests that human intelligence is more related to how efficiently the global connections of our brain are organized and how efficiently information can be integrated globally between the different regions of the brain network.

Strong associations between full-scale IQ and individual normalized path length were found in medial prefrontal gyrus, precuneus/posterior cingulate gyrus, and bilateral inferior parietal regions (Fig. 3). Recently, these regions have been reported to form functional hubs within the cortical brain network (Buckner et al., 2009). This is important, since efficient hub nodes are more likely to have a stronger effect on global network efficiency than less connected peripheral nodes. Interestingly, these frontal and parietal regions overlap the oftenreported functional default mode network (Raichle et al., 2001; Greicius et al., 2003; Damoiseaux et al., 2006; Buckner et al., 2008; van den Heuvel et al., 2008c, 2009), a dynamic resting-state network that is suggested to play a key role in processes of human cognition, like the integration of cognitive and emotional processes (Greicius et al., 2003), monitoring the world around us (Gusnard et al., 2001), and mind wandering (Mason et al., 2007). Furthermore, also the structural dynamics of these parietal and frontal brain regions have been previously linked to intelligence (Haier et al., 2004; Colom et al., 2006; Hulshoff Pol et al., 2006; Shaw et al., 2006; Jung and Haier, 2007). In addition, strong associations between normalized path length and full-scale IQ were found in left superior temporal and inferior frontal gyrus, regions that are known to play a key role in language processing (Fig. 3).

To further examine the association between $\lambda$ and IQ, a post hoc analysis was performed in which we examined the association between normalized characteristic path length $\lambda$ and the subscales of the WAIS test (Tables 1,2 ). $\lambda$ was found to be mostly related to PIQ and perceptual organization index (POI) subscores (linear regression, PIQ: $r=-0.63, p=0.004, \mathrm{df}=18$, POI: 
$r=-0.50, p=0.029, \mathrm{df}=18$ ) (supplemental Fig. 4, available at www.jneurosci.org as supplemental material). Furthermore, a second exploratory post hoc analysis was performed, examining the association between normalized path length of each individual node and IQ subscores. Strongest regional effects between IQ subscales and normalized path length were found for PIQ and POI scores (linear regression, $r<-0.4, p<0.05$ uncorrected, $\mathrm{df}=18$ ) (supplemental Fig. 5, available at www.jneurosci.org as supplemental material).

Interestingly, graph analysis of largescale anatomical networks of the primate cerebral cortex has shown before that long-range pathways play a crucial role for maintaining short processing paths across the network (Kaiser and Hilgetag, 2006). Short path length promotes high computational efficiency and is thus likely to be an important factor, together with wiring minimization (Chklovskii et al., 2002; Chen et al., 2006), for shaping cortical connectivity (Kaiser and Hilgetag, 2006). Our study may add to this discussion, indicating that a short path length is crucial in efficient global information processing and leading toward a high IQ. This suggests that functional brain networks are optimized toward processing speed and a high level of efficient global information integration between cortical and subcortical regions of the brain network.

A recent study has also shown a direct link between the structural connectivity architecture of the brain network and intellectual performance, reporting on a positive association between the microstructural organization of white matter and intelligence (Chiang et al., 2009). Among other white matter tracts, the white matter organization of the cingulum tract (as reflected by the level of fractional anisotropy measured with diffusion tensor imaging) was found to be positively associated with IQ. Interestingly, the cingulum bundle has been suggested to play an important role in structurally interconnecting the functionally linked medial frontal cortex and precuneus regions of the default mode network (van den Heuvel et al., 2008c; Greicius et al., 2009). Our current results, showing a negative association between the normalized path length of these regions and IQ (Fig. 3), suggest that in addition to the level of structural connectivity, also the level of functional communication efficiency of these regions is related to intellectual performance. Furthermore, white matter organization was found to be most prominently related to PIQ and POI subscales (Chiang et al., 2009). This supports our findings, showing a strong negative association between $\lambda$ and PIQ and POI subscores (supplemental Figs. 4, 5, available at www.jneurosci. org as supplemental material). In addition, common genetic factors were found to mediate the association between IQ and structural connectivity (Chiang et al., 2009), indicating a genetic contribution to white matter (Hulshoff Pol et al., 2006). Recent studies have suggested a high level of heritability of the levels of both the local and global interconnectedness of the brain network intelligence. characteristic path length lambda - IQ

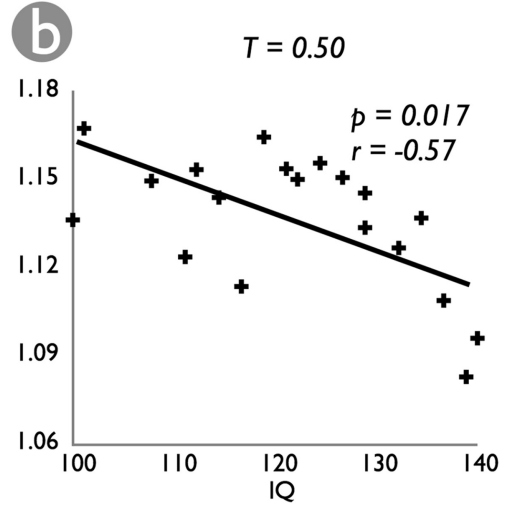

clustering-coefficient gamma - IQ

$T=0.45$

$r=-0.54$
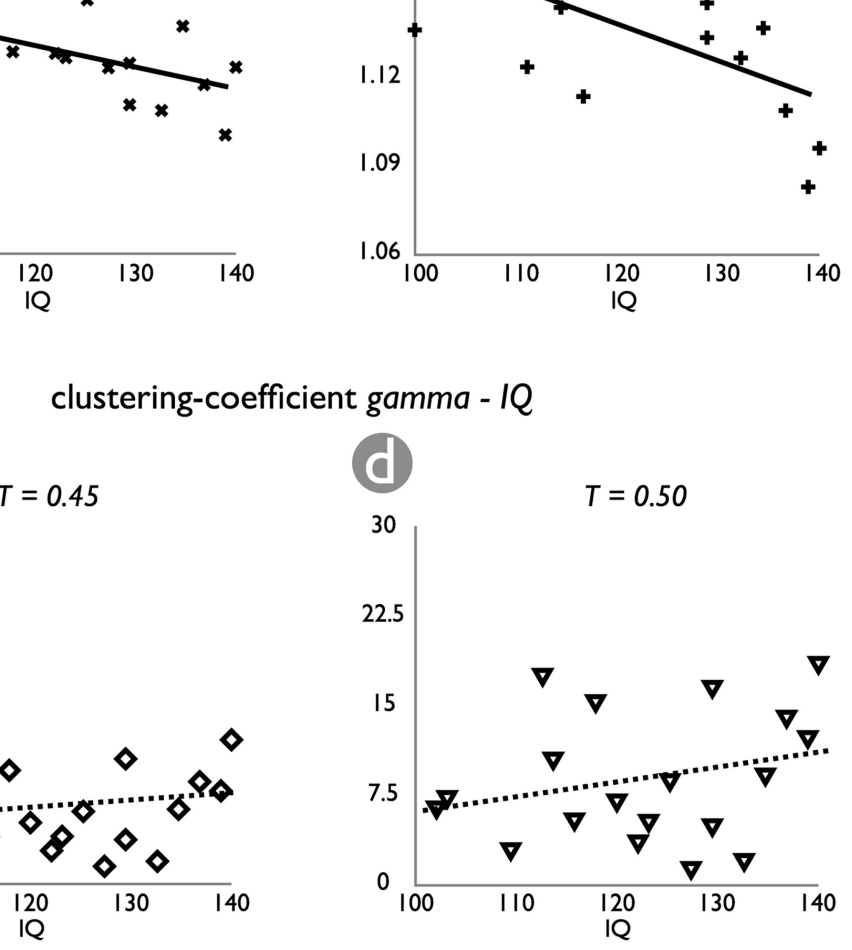

$p=0.010$

Figure 2. For networks within the small-world range $(T=0.45, T=0.50)$, the normalized characteristic path length $\lambda$ was found to be negatively correlated with $\mathrm{IQ}$, as shown in $\boldsymbol{a}$ and $\boldsymbol{b}$ [linear regression, $\mathrm{df}=18 ; T=0.45, r=-0.54, p=0.010(\boldsymbol{a})$; $T=0.5, r=-0.57, p=0.017(\boldsymbol{b})$, corrected for age]. IQ was not significantly associated with the normalized clustering suggests a strong positive association between the level of global communication efficiency within the brain network and

(Smit et al., 2008). Therefore future studies are needed to examine whether common genes mediate the association between functional and structural brain network organization and intelligence. However, although the brain's structural and functional networks are certainly linked, their exact relationship remains unclear (Bullmore and Sporns, 2009). Indeed, the reported positive association between the white matter fractional anisotropy of the posterior thalamic radiation and intelligence (Chiang et al., 2009) was not reflected in the level of functional communication efficiency of the thalamus in our study.

It is interesting to note that the reported correlations between network measures and IQ are with respect to resting-state functional connectivity and not to task-related connectivity. Our data reflects the level of efficient organization of the functional brain network during a resting state and not the efficiency of functional connectivity between brain regions during the performance of specific cognitive tasks that enter into the IQ score. As such, our data suggest that the efficiency of intrinsic resting-state functional connectivity patterns is predictive of cognitive performance.

We examined the existence of a relationship between the efficient organization of the brain network and human intelligence. Our results suggest that a short path length is crucial for efficient information processing across the functional brain network and 


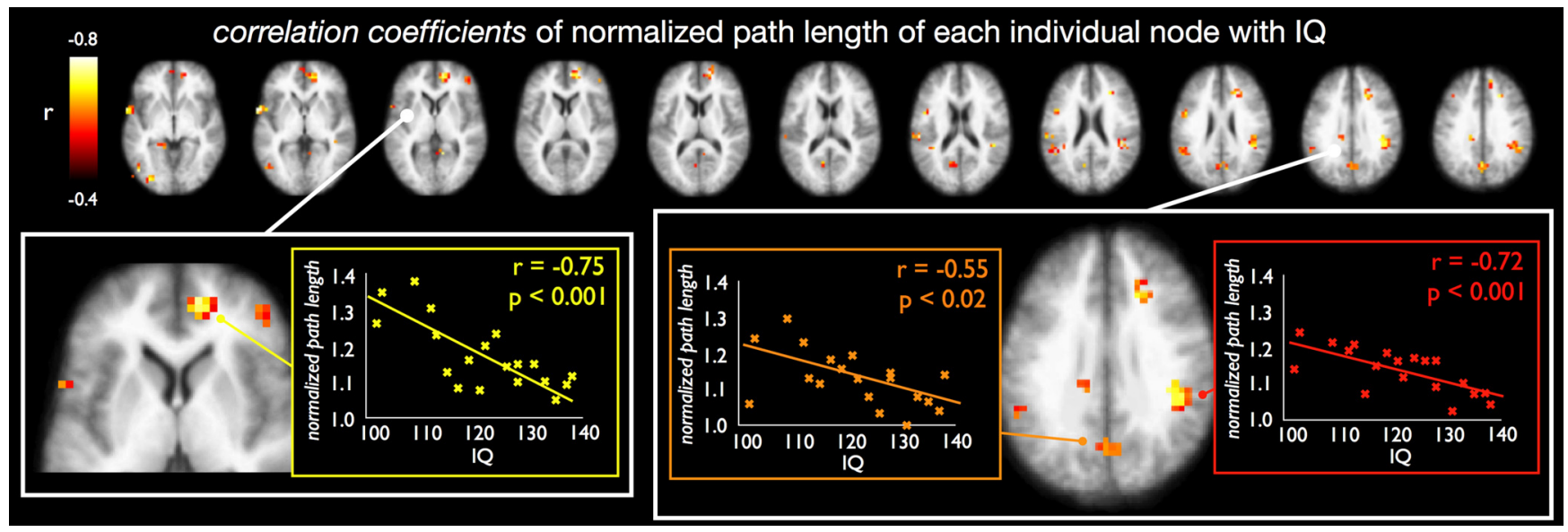

Figure 3. Most prominent effects between IQ and the level of global connectivity efficiency (as expressed by a shorter node specific normalized path length) were found in the medial prefrontal cortex (yellow box), bilateral inferior parietal cortex (red box depicts effect in right hemisphere), and precuneus/posterior cingulate regions (orange box) of the functional brain network. Shown are correlation coefficient values of those voxels that had a significant negative association between IQ and normalized path length for $T=0.45$ (linear regression, $p<0.05$ uncorrected for multiple comparisons, $\mathrm{df}=18$, corrected for age).

leading toward a high IQ. Our findings suggest a strong positive association between the efficiency of functional brain networks and intellectual performance.

\section{References}

Achard S, Salvador R, Whitcher B, Suckling J, Bullmore E (2006) A resilient, low-frequency, small-world human brain functional network with highly connected association cortical hubs. J Neurosci 26:63-72.

Aertsen AM, Gerstein GL, Habib MK, Palm G (1989) Dynamics of neuronal firing correlation: modulation of "effective connectivity". J Neurophysiol 61:900-917

Biswal B, Yetkin FZ, Haughton VM, Hyde JS (1995) Functional connectivity in the motor cortex of resting human brain using echo-planar MRI. Magn Reson Med 34:537-541.

Buckner RL, Andrews-Hanna JR, Schacter DL (2008) The brain's default network: anatomy, function, and relevance to disease. Ann N Y Acad Sci 1124:1-38.

Buckner RL, Sepulcre J, Talukdar T, Krienen FM, Liu H, Hedden T, AndrewsHanna JR, Sperling RA, Johnson KA (2009) Cortical hubs revealed by intrinsic functional connectivity: mapping, assessment of stability, and relation to Alzheimer's disease. J Neurosci 29:1860-1873.

Bullmore E, Sporns O (2009) Complex brain networks: graph theoretical analysis of structural and functional systems. Nat Rev Neurosci 10:186-198

Chen BL, Hall DH, Chklovskii DB (2006) Wiring optimization can relate neuronal structure and function. Proc Natl Acad Sci U A 103:4723-4728.

Chiang MC, Barysheva M, Shattuck DW, Lee AD, Madsen SK, Avedissian C, Klunder AD, Toga AW, McMahon KL, de Zubicaray GI, Wright MJ, Srivastava A, Balov N, Thompson PM (2009) Genetics of brain fiber architecture and intellectual performance. J Neurosci 29:2212-2224.

Chklovskii DB, Schikorski T, Stevens CF (2002) Wiring optimization in cortical circuits. Neuron 34:341-347.

Choi YY, Shamosh NA, Cho SH, DeYoung CG, Lee MJ, Lee JM, Kim SI, Cho ZH, Kim K, Gray JR, Lee KH (2008) Multiple bases of human intelligence revealed by cortical thickness and neural activation. J Neurosci 28:10323-10329.

Colom R, Jung RE, Haier RJ (2006) Distributed brain sites for the g-factor of intelligence. Neuroimage 31:1359-1365.

Damoiseaux JS, Rombouts SA, Barkhof F, Scheltens P, Stam CJ, Smith SM, Beckmann CF (2006) Consistent resting-state networks across healthy subjects. Proc Natl Acad Sci U S A 103:13848-13853.

Duncan J, Seitz RJ, Kolodny J, Bor D, Herzog H, Ahmed A, Newell FN, Emslie H (2000) A neural basis for general intelligence. Science 289:457-460.

Eguíluz VM, Chialvo DR, Cecchi GA, Baliki M, Apkarian AV (2005) Scalefree brain functional networks. Phys Rev Lett 94:018102.

Gray JR, Chabris CF, Braver TS (2003) Neural mechanisms of general fluid intelligence. Nat Neurosci 6:316-322.
Greicius MD, Krasnow B, Reiss AL, Menon V (2003) Functional connectivity in the resting brain: a network analysis of the default mode hypothesis. Proc Natl Acad Sci U S A 100:253-258.

Greicius MD, Supekar K, Menon V, Dougherty RF (2009) Resting-state functional connectivity reflects structural connectivity in the default mode network. Cereb Cortex 19:72-78.

Gusnard DA, Raichle ME, Raichle ME (2001) Searching for a baseline: functional imaging and the resting human brain. Nat Rev Neurosci 2:685-694.

Hagmann P, Cammoun L, Gigandet X, Meuli R, Honey CJ, Wedeen VJ, Sporns O (2008) Mapping the structural core of human cerebral cortex PLoS Biol 6:e159.

Haier RJ, Jung RE, Yeo RA, Head K, Alkire MT (2004) Structural brain variation and general intelligence. Neuroimage 23:425-433.

Honey CJ, Sporns O, Cammoun L, Gigandet X, Thiran JP, Meuli R, Hagmann P (2009) Predicting human resting-state functional connectivity from structural connectivity. Proc Natl Acad Sci U S A 106:2035-2040.

Hulshoff Pol HE, Schnack HG, Posthuma D, Mandl RC, Baaré WF, van Oel C, van Haren NE, Collins DL, Evans AC, Amunts K, Bürgel U, Zilles K, de Geus E, Boomsma DI, Kahn RS (2006) Genetic contributions to human brain morphology and intelligence. J Neurosci 26:10235-10242.

Jung RE, Haier RJ (2007) The parieto-frontal integration theory (P-FIT) of intelligence: converging neuroimaging evidence. Behav Brain Sci 30:135154; discussion 154-187.

Kaiser M, Hilgetag CC (2006) Nonoptimal component placement, but short processing paths, due to long-distance projections in neural systems. PLoS Comput Biol 2:e95.

Latora V, Marchiori M (2001) Efficient behavior of small-world networks. Phys Rev Lett 87:198701.

Mason MF, Norton MI, Van Horn JD, Wegner DM, Grafton ST, Macrae CN (2007) Wandering minds: the default network and stimulusindependent thought. Science 315:393-395.

Raichle ME, MacLeod AM, Snyder AZ, Powers WJ, Gusnard DA, Shulman GL (2001) A default mode of brain function. Proc Natl Acad Sci U S A 98:676-682.

Salvador R, Suckling J, Coleman MR, Pickard JD, Menon D, Bullmore E (2005) Neurophysiological architecture of functional magnetic resonance images of human brain. Cereb Cortex 15:1332-1342.

Shaw P, Greenstein D, Lerch J, Clasen L, Lenroot R, Gogtay N, Evans A, Rapoport J, Giedd J (2006) Intellectual ability and cortical development in children and adolescents. Nature 440:676-679.

Smit DJ, Stam CJ, Posthuma D, Boomsma DI, de Geus EJ (2008) Heritability of "small-world" networks in the brain: a graph theoretical analysis of restingstate EEG functional connectivity. Hum Brain Mapp 29:1368-1378.

Song M, Zhou Y, Li J, Liu Y, Tian L, Yu C, Jiang T (2008) Brain spontaneous functional connectivity and intelligence. Neuroimage 41:1168-1176.

Sporns O, Tononi G, Edelman GM (2000) Connectivity and complexity: the relationship between neuroanatomy and brain dynamics. Neural Netw 13:909-922. 
Sporns O, Chialvo DR, Kaiser M, Hilgetag CC (2004) Organization, development and function of complex brain networks. Trends Cogn Sci 8:418-425.

Stam CJ (2004) Functional connectivity patterns of human magnetoencephalographic recordings: a 'small-world' network? Neurosci Lett 355:25-28.

Stam CJ, Reijneveld JC (2007) Graph theoretical analysis of complex networks in the brain. Nonlinear Biomed Phys 1:3.

Thompson PM, Cannon TD, Narr KL, van Erp T, Poutanen VP, Huttunen M, Lönnqvist J, Standertskjöld-Nordenstam CG, Kaprio J, Khaledy M, Dail R, Zoumalan CI, Toga AW (2001) Genetic influences on brain structure. Nat Neurosci 4:1253-1258.

van den Heuvel M, Mandl R, Hulshoff Pol H (2008a) Normalized group clustering of resting-state fMRI data. PLoS ONE 3:e2001. van den Heuvel MP, Stam CJ, Boersma M, Hulshoff Pol HE (2008b) Smallworld and scale-free organization of voxel based resting-state functional connectivity in the human brain. Neuroimage 43:528-539.

van den Heuvel M, Mandl R, Luigjes J, Hulshoff Pol H (2008c) Microstructural organization of the cingulum tract and the level of default mode functional connectivity. J Neurosci 28:10844-10851.

van den Heuvel MP, Mandl RCW, Kahn RS, Hulshoff Pol HE (2009) Functionally linked resting-state networks reflect the underlying structural connectivity architecture of the human brain. Hum Brain Mapp. Advance online publication. Retrieved May 13, 2009. doi:10.1002/hbm.20737.

Watts DJ, Strogatz SH (1998) Collective dynamics of 'small-world' networks. Nature 393:440-442. 